\title{
Eficiência técnica e ciclo de vida em cooperativas: uma análise de dados em painel
}

\author{
Technical efficiency and cooperatives life cycle: a panel data analysis
}

\begin{abstract}
Resumo
O trabalho busca investigar o grau de eficiência técnica das cooperativas brasileiras através do estudo empírico das proposições da teoria do ciclo de vida, verificando assim, se a ineficiência é algo presente nas cooperativas que descontinuaram suas operações no período de 2004 a 2014. O trabalho determina o escore de eficiência técnica através da abordagem DEA (Data Envelopment Analysis). Os testes de hipótese visam a comparação entre as amostras de cooperativas descontinuadas (paralizadas, liquidadas e incorporadas) com as cooperativas que permaneceram ativas no período. Os principais resultados são que as cooperativas descontinuadas e seu subgrupo, as cooperativas liquidadas, apresentam menores níveis de receitas brutas, de folha de pagamento, despesas administrativas e ativo não circulante que as demais cooperativas. As cooperativas incorporadas têm o mesmo porte que as demais, porém são mais endividadas que elas. E por fim, validando a hipótese central do trabalho, as cooperativas descontinuadas se mostraram mais ineficientes.
\end{abstract}

Palavras-chave: eficiência técnica; cooperativismo; ciclo de vida em cooperativas; liquidação, dea.

\begin{abstract}
This paper investigates the degree of technical efficiency of Brazilian cooperatives through the empirical study of the propositions of cooperatives life cycle theory, thus verifying if inefficiency is present in cooperatives that discontinued their operations in the period from 2004 to 2014. The work determines the technical efficiency score through the Data Envelopment Analysis (DEA) approach. The hypothesis tests aim to compare the samples of discontinued cooperatives (paralyzed, settled and incorporated) with the cooperatives that remained active in the period. The main results are that the discontinued cooperatives and their subgroup, the settled cooperatives, have lower levels of gross revenues, payroll, administrative expenses and non-current assets than the other cooperatives. Incorporated cooperatives have the same size as the others, but are more indebted than them. And finally, by validating the central hypothesis of the work, the discontinued cooperatives were more inefficient.
\end{abstract}

Keywords: technical efficiency, cooperativism, cooperatives life cycle, bankruptcy, dea.

Davi Rogério Moura Costa ${ }^{\mathrm{I}}$, Lucas Batista dos Santos ${ }^{\mathrm{II}}$

${ }^{\text {I } F a c u l d a d e ~ d e ~ E c o n o m i a, ~ A d m i n i s t r a c ̧ a ̃ o ~ e ~ C o n t a b i l i d a d e ~ d e ~ R i b e i r a ̃ o ~ P r e t o ~-~ U n i v e r s i d a d e ~ d e ~ S a ̃ o ~ P a u l o ~(U S P), ~}$ Ribeirão Preto, SP, drmouracosta@gmail.com

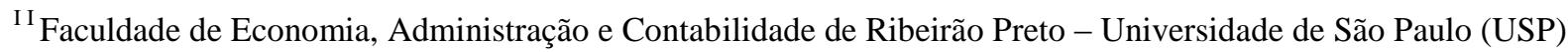

Ribeirão Preto, SP, lucas.batista.santos@ usp.br 


\section{Introdução}

Nos últimos anos as cooperativas estão se extinguindo a ritmos expressivos. A liquidação da cooperativa Cooagri, a incorporação da cooperativa Coagel pela Coamo, a aquisição da marca Paulista pela "gigante" francesa Danone, o crescente movimento de incorporações nos estados de São Paulo e Minas Gerais promovido pelas cooperativas de grande porte como a Coopercitrus (REVISTA CAFEICULTURA, 2016; JORNAL GAZETA DO POVO, 2016; REVISTA GLOBO RURAL, 2016; JORNAL VALOR ECONÔMICO, 2016) cria um cenário que intriga e motiva o meio acadêmico a se perguntar quais são os determinantes da extinção das cooperativas.

Diversos textos como Cook (1995), Hind (1999) e Cook e Burress (2009), abordam a ideia de que existe um ciclo de vida das cooperativas, onde estas instituições são criadas a partir de uma motivação econômica e posteriormente - devido a sua estrutura de direitos de propriedade e doutrinas que constituem a essência do cooperativismo - os cooperados sentem-se forçados a decidir sobre o futuro da organização quando a demanda existente no processo de criação já não é mais satisfeita ou necessária.

Segundo Chaddad e Cook (2004) a literatura ainda possui dificuldades em estabelecer uma relação entre a dissolução da cooperativa e os escores de eficiência, visto que não há muitos trabalhos que abordam o tema se utilizando de dados em painel, que acompanhem o comportamento da cooperativa por um período expressivo.

O que a literatura vem evidenciando é a desmutualização da entidade sendo afetada pela má gestão, por efeitos exógenos a cooperativa como oscilações de mercado ou mudanças de tecnologia, de modo que afetem a demanda pelos produtos da cooperativa e efeitos aliados a preferência dos próprios cooperados: a medida que a sociedade se expande e adquire novos cooperados, aumenta-se a heterogeneidade da mesma o que afeta o sentimento de "patronagem", os impulsiona a buscarem formas de organizações econômicas mais eficientes, de direitos de propriedade mais definidos, que objetivem o ganho pecuniário, como por exemplo, as sociedades anônimas (FULTON; HUETH, 2009; BURRESS; COOK, 2009).

Diante disto o objetivo deste trabalho é ampliar o estudo sobre eficiência nestas organizações por meio da investigação empírica das proposições da teoria do ciclo de vida em cooperativas através da mensuração da eficiência técnica. Isto demandará que seja mensurado o grau de eficiência destas cooperativas e verifique sua relação entre a liquidação da cooperativa e sua ineficiência.

Adicionalmente, o trabalho investigará o comportamento das variáveis utilizadas na determinação do escore de eficiência e investigará através de testes de hipótese os fatores os quais contribuíram na decisão de liquidação de cooperativas à luz da teoria do ciclo de vida. Além desta introdução este relatório contém mais quatro partes: referencial teórico, metodologia, resultados e conclusões.

\section{Referencial teórico}

A cooperativa é uma organização de propriedade do usuário, que está sob seu controle. Este também é o principal beneficiário das atividades geradas (BARTON, 1989). Desta maneira, pode-se enxergá-la, primeiramente, como uma coalizão de indivíduos a fim de, por exemplo, obter balanceamento de forças dentro de um mercado não competitivo, corrigindo as possíveis falhas existentes (STAATZ, 1987). Olhando por outro ângulo, a cooperativa também pode ser vista como um nexo de contratos ou uma extensão da propriedade do cooperado através da integração vertical (SEXTON; ISKOW, 1988). 
Para conceituar Integração Vertical deve-se pensar no processo produtivo, que tem início na extração do minério, ou no cultivo da cultura, posteriormente vai para a fábrica onde há o processamento e que acaba com o produto pronto no varejo. Todas estas etapas estão interligadas. Então quando algum grupo de produtores agrícolas decide produzir suas sementes, estão fazendo o movimento upstream (para o topo do processo), do mesmo modo quando decidem processá-lo (o seu produto) movimentam-se em sentido à downstream. Por isso através do conceito de Integração Vertical dizemos que a cooperativa é uma extensão da propriedade do cooperado (SEXTON; ISKOW, 1988).

A criação das cooperativas agropecuárias é decorrente da motivação de um grupo de produtores a fim de melhorar sua posição socioeconômica diante de uma situação de falha de mercado (COOK; BURRESS, 2009; STAATZ, 1987). Dessa maneira, a reparação de falhas de mercado - extinção do poder de mercado (monopólio/monopsônio) e da falta de informação entre compradores e vendedores (caso de assimetria de informação) - permite a inserção dos produtores num mercado não competitivo (COOK; BURRESS, 2009).

As melhorias da condição socioeconômica dos produtores associados gera externalidades positivas. Assim, o Estado em geral cria políticas de incentivo à criação destas organizações através de isenções e subsídios (HANSMANN, 1996).

Cook (1995) aponta que existe um ciclo de vida das cooperativas. De acordo com o autor, há cinco fases. A primeira foi introduzida acima como Justificativa Econômica. A segunda é onde ocorre a estruturação da sociedade. Há a separação entre a propriedade e a gestão da cooperativa. Costa (2010) afirma que é dado a um agente o direito de controle formal da organização. Esse agente é contratado por uma diretoria eleita pelos detentores dos direitos residuais da firma em uma Assembleia Geral. É eleito também um Conselho Administrativo. Este tem o papel de ratificar e monitorar os projetos propostos e iniciados pela gestão (CHADDAD; ILIOPOULOS, 2013).

A terceira fase é chamada de Crescimento, Glória e Heterogeneidade. Trata-se do período em que a cooperativa se expande até atingir sua máxima, em termos de saúde financeira (COOK; BURRESS, 2009). Nesta fase problemas de governança corporativa são acentuados devido à limitada definição de direitos de propriedade e elevados custos de agência, afetando negativamente a eficiência da organização (FERREIRA, 2005).

Braga, Ferreira e De Souza (2011) apresentam três problemas de governança corporativa que influenciam na eficiência das cooperativas, o "problema do carona" que é o comportamento oportunista do cooperado que tem direito ao benefício que os demais cooperados sem terem tido o mesmo nível de investimento que os demais. O segundo "problema é o horizonte", devido ao curto horizonte de planejamento, gerado pela intransferibilidade das quotas-parte. Como há fluxo de associados, a cooperativa tem dificuldade de se projetar no longo prazo, pois quando o cooperado deixá-la levará consigo o capital investido na sua entrada a cooperativa. E o terceiro é o "problema do portfólio", as cooperativas não são economicamente racionais porque não decidem diversificar seus produtos com o fim em garantir sua perpetuidade no mercado. As cooperativas determinam os produtos que irão ofertar baseado nos interesses específicos de seus cooperados. Além desses, existe o "problema do controle".

É interessante acrescentar que segundo Nilsson (1999), as cooperativas são irracionais para a formulação de portfólio, pois não diversificam suas atividades a fim de aproveitarem oportunidades de mercado, mas para satisfazer os interesses de seus associados. A questão dos problemas de governança corporativa nas cooperativas será retomada na próxima seção. 
Na quarta fase, a cooperativa passa a colher os frutos do seu mau posicionamento estratégico. A teoria afirma que esta é uma fase onde a cooperativa está em decadência financeira e que consequentemente os cooperados iniciam um processo de reflexão acerca do futuro da mesma.

A quinta fase é chamada de escolha; onde a direção decide efetivamente o futuro da cooperativa. As opções citadas por Cook e Burress (2009) são: continuar com a mesma estratégia; reinvenção; desmutualização; e liquidação.

A reinvenção consistiria, de acordo com os autores, em mudar o portfólio, sua estrutura de governança, adequações dos direitos de propriedade, etc.; a desmutualização implica no desmembramento, por exemplo, em outras cooperativas de menor porte. Por fim, a liquidação é o encerramento das atividades.

A proposta do Ciclo de Vida em Cooperativas é interessante porque vai de encontro com o conceito da perpetuidade, um dos "dogmas" da contabilidade.

\subsection{Eficiência em Cooperativas}

Segundo Ferreira (2005) eficiência em cooperativas pode ser vista como uma combinação entre racionalidade econômica e valores de ajuda mútua, igualdade, justiça, solidariedade e liberdade. Por isso a ação cooperativista visa a construção de um empreendimento que gere, concomitantemente ao ganho pecuniário, externalidades positivas à sociedade cuja cooperativa esta inserida.

Para Pindyck e Rubinfeld (1998) eficiência é a área das ciências econômicas que se referem à alocação ótima de recursos e ausência de desperdícios com fim em obter maior produto, dado os insumos disponíveis. Então, eficiência permite a alocação de recursos a fim de atribuir a essa alocação um score e assim é possível ordenar cardinalmente as empresas considerando seus níveis de eficiência.

Os economistas ortodoxos conceituam eficiência econômica sob a ótica do ótimo de Pareto, onde eficiente é a empresa que maximiza sua função-objetivo maximizando o lucro ou minimizando os custos de produção e, para o consumidor, maximizando a satisfação ou minimizando as despesas (MILER, 1981).

Farrel (1957) conceitua uma medida de eficiência econômica que é constituída de duas componentes: técnica e alocativa. A primeira aborda a eficiência técnica e informa sobre o processo de organização dos insumos para a obtenção do máximo de produto; o segundo aponta a forma como os insumos são utilizados em proporções ótimas, dado seus preços relativos.

Para Ferreira (2005) eficiência econômica é descrita conforme equação (1) abaixo:

$$
E E=E T x E A(1)
$$

E a partir da mensuração do escore de eficiência técnica é possível calcular o escore de eficiência de escala o qual informa o quão ideal é a escala em que a DMU (Decision Making Unit, no caso em questão, a cooperativa) está operando. Na metodologia do trabalho será explicada a forma de cálculo ${ }^{1}$.

As organizações cooperativas possuem peculiaridades que são relevantes para o cálculo de eficiência técnica, elas são organizações econômicas que possuem direitos de propriedade vagamente 
definidos (BARTON, 1989). Isto contribui para a criação de problemas de governança que culminam em ineficiências.

Porter e Scully (1987) afirmam que a estrutura de direitos de propriedade e o cenário constitucional implicam em ineficiências estruturais que não podem ser corrigidos nas cooperativas.

A primeira delas decorre da existência do "problema do horizonte", que surge porque os direitos de propriedade não são comercializáveis. A heterogeneidade dos associados contribui para expectativas de investimentos distintas. Esse fator é agravado pelo fato do cooperado, quando deixar a cooperativa, resgatar somente o montante investido, então os incentivos ao investimento na cooperativa são quase nulos (ZILBERSZTAJN, 2002; COOK; ILIOPOUOLOS, 1998). Isto reflete na baixa capacidade de autofinanciamento das cooperativas e na acentuada utilização de capital de terceiros (SOUZA; BRAGA; FERREIRA, 2011).

A segunda surge do "problema do carona" que descreve um comportamento oportunista por parte do cooperado que usufrui de benefícios que a cooperativa oferece, mas não arca totalmente com o custo deste. Um efeito deste problema é que as cooperativas recebem cada vez mais volumes de mercadorias, no entanto não possuem infraestrutura para processá-los, pois não recebem investimentos suficientes para sua ampliação, gerando assim, maiores volumes de estoques e superutilização da capacidade produtiva (SOUZA; BRAGA; FERREIRA, 2011).

O terceiro fator de ineficiência decorre da existência do "problema do portfólio". Como dito no referencial teórico, as cooperativas são irracionais para a formulação de portfólio, pois não diversificam suas atividades a fim de aproveitarem oportunidades de mercado, mas para satisfazer os interesses de seus associados. Isto implica em investimentos pobres que, mais tarde, culminam em ineficiências alocativas (EVANS; GUTHRIE, 2006). O quarto decorre do "problema do controle".

Para o este problema, Costa (2010) destaca três diferentes prismas na literatura sobre cooperativas. O primeiro abordado por Jensen e Meckling (1979) que acredita ser danosa para a organização a participação dos proprietários na gestão da cooperativa, uma segunda linha teórica aponta que é benéfica a participação dos cooperados no controle da organização, pois o profissional contratado tomaria decisões que reduzem o valor dos direitos ao resíduo dos cooperados (VITALIANO, 1983). E um ponto de vista neutro estabelece que a participação dos cooperados no controle contribua com a visão estratégica dos negócios, uma vez que conhecem o principal negócio da cooperativa. Porém, à medida que a organização se torna complexa a sua participação pode tornar a gestão empobrecida (STAATZ, 1987; HELMBERGER 1966). O último ponto a ser analisado são os "custos de influencia" trata-se das ineficiências provocadas por decisões que visam interesses específicos de seus associados. A leitura de Zylbersztajn (2002) e Bialoskorski Neto (2012) é essencial para o aprofundamento no tema.

Em síntese, Porter e Scully (1987) afirmam que a estrutura de direitos de propriedade geram ineficiências estruturais que não podem ser corrigidas. Fatores como a não comercialização das quotas e a distribuição das sobras através do uso e a característica das decisões pelo "um homem um voto", culminam em problemas de governança corporativa como a questão do "carona", do "horizonte" e custos de agência como o custo de influencia (BIALOSKORSKI NETO, 2012; SOUZA; BRAGA; FERREIRA, 2011; COOK; ILIOPOUOLOS, 1998; COSTA, 2010; FERREIRA,2005; HANSMANN, 1996; NILSSON, 1999; ZILBERSZTAJN, 2002).

Assim, é necessário ponderar a eficiência pelas ineficiências decorrentes das características dos direitos de propriedade das cooperativas. Caso contrário o escore de eficiência técnica gerada será 
sempre subestimado. E isto se torna crucial para efeito de comparação entre cooperativas e empresas "convencionais".

\section{Metodologia e descrição da amostra}

O estudo foi feito com dados cedidos pelo Observatório do Cooperativismo através de uma parceria com a Organização das Cooperativas do Estado do Paraná (OCEPAR) e Organização das Cooperativas Brasileiras no Mato Grosso do Sul (OCB-MS). A base de dados contém 138 cooperativas que existiram em algum momento de 2004 a 2014.

Por problemas de padronização das demonstrações contábeis onde algumas cooperativas não apresentaram a informação de despesa com pessoal ou algo semelhante destacado na DRE (Demonstração do Resultado do Exercício), a base foi reduzida para 103 cooperativas.

Foi possível calcular os escores de eficiência técnica para cada uma delas. Do total, oitenta cooperativas ainda estavam em operação, seis estavam na condição de liquidadas, sete tinham sido incorporadas e dez estavam em situação de paralisação, isto é, não estavam funcionando.

Na tabela 1 é apontada a estatística descritiva das cooperativas, considerando seus status de funcionamento. Esta tabela nos dá a noção de tamanho das cooperativas trabalhadas.

Note na tabela que há um grau alto de heterogeneidade vista por seu desvio padrão, principalmente quando é falado sobre as cooperativas ativas. Isto expressa que a amostra condiz com a realidade

Tabela 1 - Estatística descritiva da amostra, considerando o ativo e passivo sobre patrimônio líquido das cooperativas de 2004 a 2014.

\begin{tabular}{|c|c|c|c|c|c|}
\hline Status & Variável & Média & $\mathrm{DP} * *$ & Mínimo & Máximo \\
\hline \multirow{2}{*}{ Ativa } & Ativo (R\$ - mil) & $551.251,80$ & 1.055 .235 & 139,16 & $7.024 .264,00$ \\
\hline & Endividamento & 1,98 & 3 & $-5,60$ & 32,98 \\
\hline \multirow{2}{*}{ Incorporada } & Ativo (R\$ - mil) & $391.160,00$ & 423.247 & 1107,85 & $1.305 .185,00$ \\
\hline & Endividamento & 6,37 & 6 & 0,07 & 17,66 \\
\hline \multirow{2}{*}{ Liquidada } & Ativo (R\$ - mil) & $19.408,43$ & 22.347 & 1187,65 & $99.642,73$ \\
\hline & Endividamento & 0,64 & 8 & $-26,79$ & 33,91 \\
\hline \multirow{2}{*}{ Paralisada } & Ativo (R\$ - mil) & $108.129,50$ & 221.327 & 208,08 & $774.063,10$ \\
\hline & Endividamento & 3,82 & 12 & $-48,59$ & 78,90 \\
\hline
\end{tabular}

Fonte: Elaboração própria.

Nota: ** Desvio-Padrão (DP) 
O Ativo Total das cooperativas liquidadas e paralisadas se apresentou menor que o Ativo Total das cooperativas Ativas e Incorporadas, claro que, é necessário fazer os testes de hipóteses para efetuar qualquer tipo de afirmação. No entanto, este comportamento fomenta a indagação sobre a contraposição dos ativos entre as cooperativas liquidadas e incorporadas.

Seguindo o mesmo raciocínio, pode-se ver que há cooperativas que possuem Patrimônio Líquido Negativo $^{2}$ entre as cooperativas ativas e Liquidadas. Este é um ponto importante, pois isto indica que estas estavam insolventes. De acordo com a teoria, a cooperativa pode entrar em dissolução por má gestão (FULTON; HUETH, 2009), então as cooperativas ativas que apresentam o saldo do Patrimônio Líquido negativo podem ser candidatas a liquidadas no futuro. E em contraposição as cooperativas incorporadas não possuíam Patrimônio Líquido negativo, mas apresentaram, em média, Passivos 6,37 vezes maiores que o Patrimônio Líquido. Por isso talvez cooperativas incorporadoras tendam a não buscar cooperativas com Patrimônio Líquido negativo.

Estas afirmações ainda não foram testadas por isso são somente inferências a partir dos valores apresentados nesta tabela.

\subsection{Mensuração de eficiência}

Existem vários métodos de mensuração da eficiência. Neste trabalho decidiu-se utilizar o método de Análise por Envelopamento de Dados (DEA), com a utilização do software STATA 13.1 para os cálculos. A motivação para sua adoção consistiu no fato do método não demandar nenhum tipo de estimativa estatística da função produção das empresas analisadas.

A DEA é um método não paramétrico de mensuração de eficiência que consiste na aplicação de técnicas de programação linear, largamente empregadas no planejamento de operações empresariais e de políticas públicas, a avaliação da gestão dessa política pública (CHARNES; COOPER; RHODES, 1981; PRIOR; SOLÀ, 2001). A técnica mede a distância de cada Decision Making Unit (DMU) da fronteira de eficiência, atribuindo à DMU um escore situado entre zero e um.

Uma vantagem na utilização desse modelo em relação aos modelos de mensuração de eficiência paramétricos se dá em não necessitar de nenhum tipo de estimativa estatística da fronteira de produção da DMU. E sua característica multi-insumo e multiproduto, permitindo a atribuição de dados não monetários no cálculo. E sua limitação está na impossibilidade de trabalhar com valores negativos e na necessidade de métodos refinados de matemática para o trabalho com dados em painel.

Ferreira (2005) afirma que a programação linear consiste em um processo que visa a maximização ou a minimização da função objetivo, dado a uma equação condicionante. $\mathrm{O}$ que em outras palavras significa a resolução de um problema de otimização de uma função sujeita a suas restrições.

Para executar os cálculos são necessários que sejam levados em conta os pressupostos de que a base de dados possua DMUs que comungam dos mesmos insumos e produtos, autônomas na tomada de decisão e homogêneas, que operam na mesma unidade de medida.

Ao longo do tempo foram feitos vários estudos que visavam aprofundar a abordagem DEA, assim foram construídos vários modelos para a mensuração da eficiência. Os modelos mais conhecidos são os modelos CCR (Charnes, Cooper E Rhodes), o qual propõe a mensuração da eficiência pressupondo retornos de escala constantes e o modelo BCC (Banker, Charnes e Cooper) que pressupõe retornos de escala variáveis. 
A forma algébrica do modelo CCR:

$$
\max _{\phi, \lambda} \phi_{,}(2)
$$

Sujeito a:

$$
\begin{gathered}
\phi y_{j}-\gamma \lambda \leq 0 \\
-x_{i}+X \lambda \leq 0 \\
-\lambda \leq 0, \lambda \text { não negativo }
\end{gathered}
$$

Onde é um vetor ( $\mathrm{a}$ x 1 ) de quantidade de produto da i-ésima DMU; , um vetor ( $\mathrm{b}$ x 1 ) de quantidade de insumos da i-ésima DMU; Y, uma matriz ( $\mathrm{n} x$ a) de produto das $\mathrm{n}$ DMUs; $\mathrm{X}$, uma matriz ( $\mathrm{n} \times \mathrm{b}$ ) de insumo das $\mathrm{n}$ DMUs; $\lambda$, um vetor de ( $\mathrm{n} \times 1$ ) pesos; $\phi$, um escalar com valores maiores ou iguais a 1 que indica o valor de eficiência técnica da i-ésima DMU em relação às demais (Ferreira e Braga 2011).

Diferentemente do modelo CCR, o modelo BCC também pode informar se a DMU está atuando em níveis de escala crescente ou decrescente (BANKER; CHARNES; COOPER, 1984).

A forma algébrica do modelo BCC é apresentada no conjunto de equações (3) abaixo:

$$
\max _{\phi, \lambda} \phi_{,}(3)
$$

Sujeito a:

$$
\begin{gathered}
\phi y_{j}-y \lambda \leq 0 \\
-X_{i}+X \lambda \leq 0 \\
\mathrm{~N}_{1} \lambda=1, \lambda \text { não negativo }
\end{gathered}
$$

As variáveis são iguais às descritas no modelo CCR com exceção de $\mathrm{N}$, um vetor $\left(\begin{array}{lll}\mathrm{N} & \mathrm{x} & 1\end{array}\right)$ identidade, composto do número um.

A eficiência de escala (ES) é a razão entre a eficiência técnica pressupondo retornos de escala constantes com o modelo CCR com orientação para o produto (ETRCE) e a eficiência técnica pressupondo retornos de escala variáveis com o modelo BCC com orientação para o produto (ETRVE).

$$
E S=\frac{E T_{R C E}\left\{\left[X_{R}, Y_{R}\right]\right.}{E T_{R V E}\left\{\left[X_{R}, Y_{R}\right\rfloor\right.}
$$


Para identificar se a DMU está atuando em retornos de escala crescentes ou decrescentes, primeiramente é preciso exercer o cálculo do modelo BCC com orientação para produto sob o pressuposto de retornos não crescentes de escala (ETRNC). Para isto é necessário alterar a restrição de $\mathrm{N}_{1} \lambda=1$ para $\mathrm{N}_{1} \lambda \leq 1$.

Assim, se:

- $\mathrm{ET}_{\mathrm{RNC}}=\mathrm{ET}_{\mathrm{RVE}}-\mathrm{A}$ DMU opera em retornos decrescentes de escala;

- $\mathrm{ET}_{\mathrm{RNC}} \mathrm{ET}_{\mathrm{RVE}}$ - A DMU opera em retornos crescentes de escala.

\subsubsection{Variáveis do Modelo DEA}

Para a seleção das variáveis da DEA foram levados em consideração os aspectos apontados na literatura. Assim, tem-se como variável representante do Produto $(\mathrm{Y})$ da cooperativa a receita bruta (RB), como medida para o resultado global da DMU.

Os insumos (X) foram representados pelas variáveis: gasto total com empregados (FP), para representar o fator trabalho; ativo não circulante $(\mathrm{ACN})$, como medida para o fator capital; e despesas administrativas (DA) como Proxy para a estrutura operacional da DMU.

\subsection{Testes de Hipótese}

O trabalho reuniu uma série de testes de hipótese com o objetivo de verificar se condiz com a realidade o que a teoria afirma sobre o comportamento das cooperativas que entraram em liquidação.

O que se esperava do comportamento que justifique a decisão de uma cooperativa em encerrar suas atividades é uma baixa capacidade de autofinanciamento devido a um desincentivo ao aporte de capital na mesma (BIALOSKORSKI NETO, 2012) e também, como em qualquer outra entidade, a gestão pouco efetiva. (FULTON; HUETH, 2009; CHADDAD; COOK, 2004).

A saída da cooperativa do mercado pode ser fruto também de mudanças das condições do mercado, como alterações da demanda agregada do mesmo. Porque se esta cooperativa está na margem do mercado está vulnerável a oscilações do mesmo.

Agora, pensando na fase cinco do ciclo de vida, segundo Shrader (1989) a avaliação dos ativos de uma cooperativa influencia em seu futuro como cooperativa. Ao passo que se espera que haja correlação entre a opção de liquidação (ou incorporação e afins) e seu valor.

Assim sendo, o trabalho comparou o nível de comprometimento dos recursos próprios frente ao capital de terceiros (Passivo sobre Patrimônio Líquido - ENDIVIDAMENTO) e o tamanho do ativo da cooperativa (ATIVO TOTAL). Além de comparar os escores de eficiência técnica (ETRVE), eficiência técnica pura (ETRCE) e eficiência de escala (ES) que fazem parte da proposta do trabalho.

Também foi comparado o comportamento das variáveis que compuseram a modelagem DEA para complementar a análise: receita bruta $(\mathrm{RB})$, gasto total com empregados $(\mathrm{FP})$, para representar o fator trabalho; ativo não circulante $(\mathrm{ACN})$, como medida para o fator capital; e despesas administrativas (DA).

Os testes de hipótese foram executados levando em conta erro tipo um de $5 \%$ e unilaterais a fim de dizer se o valor expresso da variável analisada com hipótese nula Ho testando se as médias 
apresentadas são iguais, H1 se a média do segundo grupo é maior hipótese $\mathrm{H}_{2}$ se são diferentes e hipótese $\mathrm{H}_{3}$ se o valor da média do segundo grupo é menor. A ordem de comparação dos grupos foi: cooperativas liquidadas com as demais; cooperativas incorporadas com as demais; foram agregadas todas as cooperativas que descontinuaram suas operações, comparando estas com as que permaneceram ativas; e por fim, as cooperativas que entraram em liquidação com as cooperativas que foram incorporadas.

\section{Resultados finais e discussões}

Na tabela 2 abaixo está apresentada a estatística descritiva das variáveis que compuseram o modelo DEA para cálculo de eficiência.

Como se pode notar, considerando todas as observações do painel, a cooperativa média da amostra tinha RB de $\mathrm{R} \$ 615.432,30$ mil e uma DA de $\mathrm{R} \$ 10.696,00$ mil, o seu ANC era de $\mathrm{R} \$ 179.018,60$ mil e FP de R\$20.191,63 mil. E os elevados valores dos desvios-padrão denunciam uma heterogeneidade das cooperativas na amostra.

Tabela 2 - Estatística Descritiva das Variáveis que compuseram o modelo DEA (R\$ - mil).

\begin{tabular}{llcll}
\hline Variável & Média & Desvio-padrão & Mínimo & Máximo \\
\hline RB & $615.432,30$ & 1276880 & 7,53 & $11.100 .000,00$ \\
\hline DA & $10.696,00$ & 22723 & 0,65 & $179 \cdot 517,70$ \\
\hline ANC & $179.018,60$ & 315257 & 0,10 & $1.965 .555,00$ \\
\hline FP & $20.191,63$ & 43625 & 10,24 & $362.280,00$ \\
\hline
\end{tabular}

Fonte: Elaboração própria.

Na tabela 3 são apresentadas as estatísticas descritivas dessas variáveis, considerando o status da cooperativa. Isto é, sua condição de funcionamento no período: ativa o período todo, incorporada, liquidada ou paralisada. Foram feitos testes de média para demonstrar se os valores apresentados nesta tabela são maiores ou menores entre si. Primeiramente, foram comparadas as amostras das cooperativas liquidadas com as demais cooperativas. 
Tabela 3 - Estatística das variáveis que compuseram o modelo DEA segregadas por seus Status (R\$ - mil).

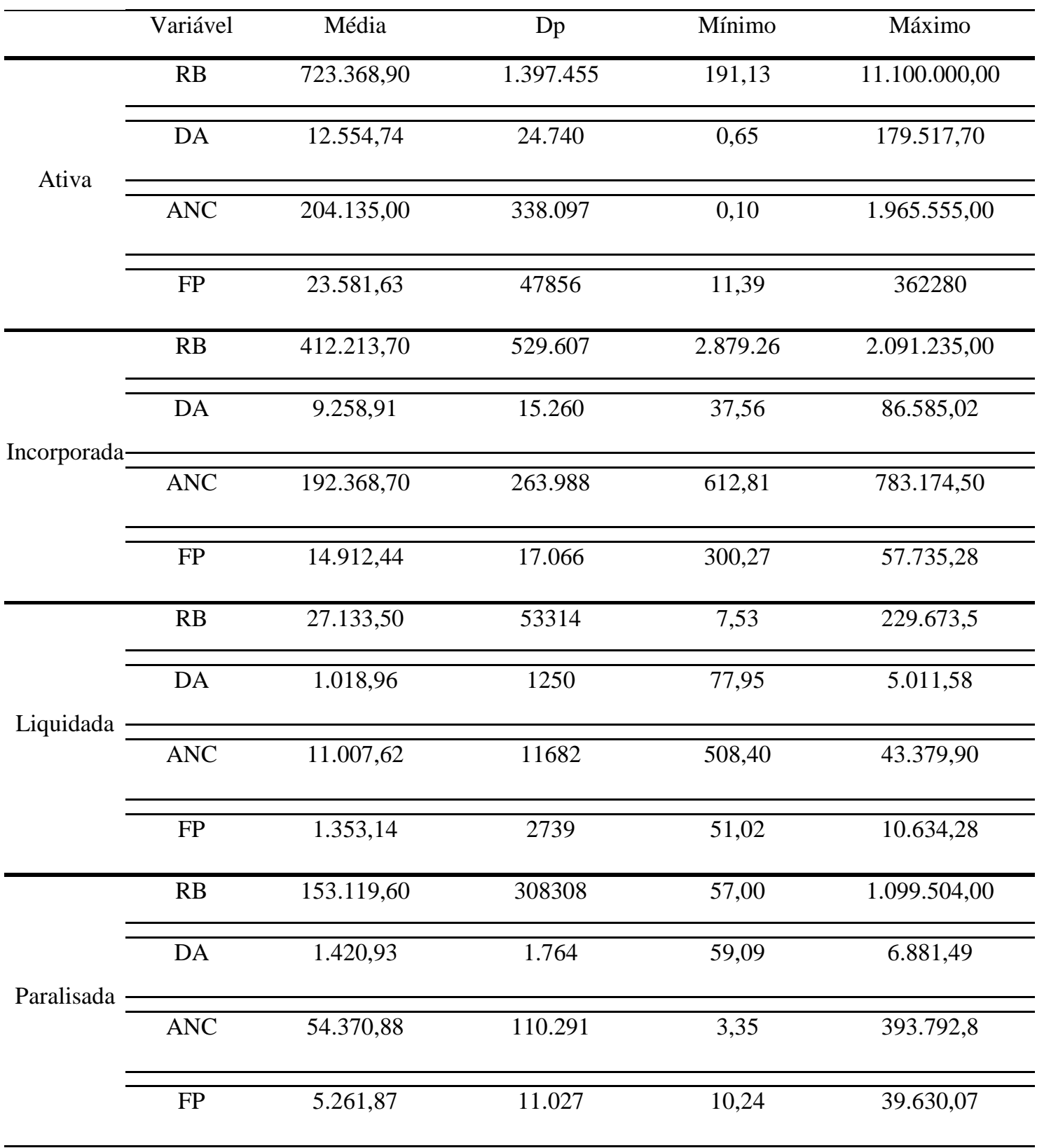

Fonte: Elaboração Própria

As receitas (RB) das cooperativas liquidadas se mostraram menores (rejeitou-se a hipótese nula de igualdade) que as demais com p-valor igual a o,0037; sobre a variável $\mathrm{FP}$, verificou-se que as cooperativas liquidadas são menores com p-valor igual a o,oo61; no que tange a variável DA os valores das liquidadas se mantiveram menores com p-valor de o,0067; e de maneira semelhante, as cooperativas liquidadas apresentaram ANC menor com p-valor de o,oo10.

Isto evidencia que as cooperativas liquidadas possuem um porte inferior a média; movimentam quantidades menores de recursos. 
A segunda comparação foi entre as cooperativas incorporadas frente às demais. Feito os testes, não foram rejeitadas as hipóteses nulas de todos os testes no que tange a RB, FP, DA e ANC com os respectivos p-valores de o,1668; o,2311; 0,3504; e o,6015. Isto é um dado importante porque demonstra que as cooperativas que são incorporadas possuem o mesmo porte que as cooperativas que se mantém vivas, o que indica que as cooperativas incorporadoras levam em consideração a quão expressiva são as operações das incorporadas antes da incorporação. Porque se presume que o montante de recursos o qual a empresa movimenta seja proporcional a sua capacidade de geração de fluxos de caixa, assim sendo, o que se evidencia a partir destes dados é que as cooperativas são igualmente "capazes" que as demais cooperativas.

Comparando as cooperativas descontinuadas com as ativas os testes de hipótese apresentaram os seguintes resultados: as cooperativas descontinuadas apresentaram índices menores de RB, DA, FP e ANC de acordo com os seguintes p-valores o,oooo; o,oooo; o,oooo; e o,oooo.

Estas características apontam que as cooperativas que optam por descontinuar suas atividades estão situadas em níveis de produção menores, em outras palavras, as cooperativas que optam por descontinuar suas atividades estão em quantis menores que as cooperativas que se mantém operando. Isto talvez sinalize que estas estavam à margem do mercado, por isso sucumbiram.

Esta afirmação deve levar em conta que a média das cooperativas incorporadas eleva a média das cooperativas descontinuadas. Por isso é importante que também sejam comparados os casos individuais.

Por fim comparando as cooperativas liquidadas com as incorporadas foi verificado que as cooperativas liquidadas demonstraram menores níveis de RB, FP, DA e ANC, com os seguintes pvalores: o,oool; o,000o; o,0017 e o,0001, respectivamente.

Este resultado deve ser visto com calma, pois evidencia o que foi citado anteriormente sobre a questão das cooperativas incorporadoras. $\mathrm{O}$ que vem sendo demonstrado com os resultados desses testes são que as menores cooperativas tendem a serem liquidadas e cooperativas maiores tendem a serem incorporadas na fase cinco do ciclo de vida.

Além dos testes de hipóteses com as variáveis apresentadas na tabela 3, foram elaborados testes de médias sobre as variáveis da tabela 1 (Ativo Total e Endividamento) no mesmo formato dos testes descritos acima, comparando as liquidadas e incorporadas com as demais cooperativas, descontinuadas com ativas e liquidadas com incorporadas.

Feito o teste de médias comparando as cooperativas liquidadas com as demais cooperativas rejeitou-se a hipótese nula de igualdade de médias entre as duas amostras para os valores de ativo apresentados com p-valor de o,o032. Semelhantemente, rejeitou-se a hipótese nula de igualdade das médias das amostras para o nível de endividamento com p-valor de o,0301.

Isso implica em uma indagação sobre o grau de endividamento das cooperativas liquidadas. Talvez o motivo da liquidação das cooperativas não seja pelo nível de endividamento, mas talvez pela capacidade de geração de fluxo de caixa das mesmas - veja que ativos menores, adicionados a menores níveis de receita culminam em uma capacidade de geração de fluxo de caixa menor.

A problemática deve ser vista com calma: a partir do momento em que a cooperativa passa a ter o Patrimônio Líquido negativo esta tem problemas de endividamento, mas este problema está mais ligado a solvência da mesma que o excesso de empréstimos. 
Portanto, o resultado acima expressa que o prejuízo acumulado dessas organizações culminou em um Patrimônio Líquido negativo e assim na sua liquidação. Veja, este é um problema de geração de fluxo de caixa, não de endividamento.

Comparando os ativos das cooperativas incorporadas com as demais, não foi rejeitada a hipótese nula com p-valor de o,3049 e rejeitou-se a hipótese nula com p-valor de o,oooo com as cooperativas apresentando nível de endividamento maior que as demais. Este dado demonstra que a incorporação da cooperativa talvez esteja mais ligada ao grau de endividamento da mesma.

As cooperativas descontinuadas apresentaram valores de ativo total inferior às demais cooperativas com p-valor de o,oooo e maior grau de endividamento com p-valor de o,ooo3. O trabalho destaca a importância de saber qual foi à decisão na fase cinco, porque quando analisado somente os dois últimos testes tem-se a impressão de que as cooperativas menores e mais endividadas tendem a sair do mercado o que não é, necessariamente, verdade.

Quando comparadas as cooperativas liquidadas e incorporadas pôde ser percebido, através do teste de hipótese, que as cooperativas liquidadas apresentaram menor ativo num p-valor de o,oooo e que as cooperativas incorporadas se mostraram mais endividadas com p-valor de o,ooo6.

Bialoskorski Neto (2012) afirma que as cooperativas têm dificuldades de autofinanciamento o que as fazem procurar recursos de terceiros. Isto é algo para se refletir por que: para concessão de crédito os bancos avaliam os ativos das empresas e sua capacidade de geração de fluxos de caixa. Assim, é de se esperar que cooperativas de menor porte não tenham tanto acesso ao crédito como as cooperativas de maior porte.

Deste modo, se há uma mudança no mercado - num caso hipotético - somada da necessidade de busca de empréstimos, as cooperativas de maior porte o conseguem, mas as cooperativas de menor porte não. E isso implica que as cooperativas de menor porte saem deste mercado, seja pela paralisação de suas atividades ou pela liquidação da mesma.

E se a gestão das cooperativas de maior porte, que adquirem empréstimos, não for eficiente em casar os fluxos de caixa, tempos depois esta será incorporada - note aí a importância de uma gestão eficiente. Na tabela 4 adiante são expostos os valores referentes aos escores médios de eficiência das cooperativas, conforme o status da organização.

Tabela 4 - Estatísticas descritivas dos escores de Eficiência Técnica, de Escala e dos Retornos de Escala no período de 2004 a 2014 por Status.

\begin{tabular}{|c|c|c|c|c|c|}
\hline Status & Variável & Média & $\begin{array}{l}\text { Desvio- } \\
\text { padrão }\end{array}$ & Mínimo & Máximo \\
\hline \multirow{4}{*}{ Ativa } & ETRCE & 0,5273 & 0,2636 & o,oo89 & 1 \\
\hline & ETRVE & 0,7041 & 0,2836 & o,oo88 & 1 \\
\hline & ES & 0,7614 & 0,1956 & 0,2958 & 1 \\
\hline & RTS & $-0,4761$ & o,8017 & -1 & 1 \\
\hline \multirow{3}{*}{ Incorporada } & ETRCE & 0,4546 & 0,2732 & 0,0150 & 1 \\
\hline & ETRVE & 0,5713 & 0,3245 & 0,0151 & 1 \\
\hline & ES & 0,8217 & 0,1832 & 0,4097 & 1 \\
\hline
\end{tabular}


93 | Eficiência técnica e ciclo de vida em cooperativas: uma análise de dados em painel

\begin{tabular}{|c|c|c|c|c|c|}
\hline & RTS & $-0,2857$ & 0,9258 & -1 & 1 \\
\hline \multirow{4}{*}{ Liquidada } & ETRCE & 0,2584 & 0,2516 & 0,0003 & 1 \\
\hline & ETRVE & 0,2988 & 0,2800 & 0,0003 & 1 \\
\hline & ES & o,9077 & 0,1240 & 0,5436 & 1 \\
\hline & RTS & $0,375^{\circ}$ & o,8706 & -1 & 1 \\
\hline \multirow{4}{*}{ Paralisada } & ETRCE & 0,5877 & 0,3091 & 0,0261 & 1 \\
\hline & ETRVE & 0,6885 & 0,3070 & 0,0261 & 1 \\
\hline & ES & 0,8556 & o,1696 & 0,3473 & 1 \\
\hline & RTS & $-0,2272$ & 0,8374 & -1 & 1 \\
\hline
\end{tabular}

Fonte: Idealização própria

Testando os valores apresentados na tabela pode-se concluir que em média os escores de eficiência técnica considerando retornos de escala constante (ETRCE) e variável (ETRVE) são menores em cooperativas liquidadas quando comparadas com as demais cooperativas, com p-valor de o,00oo e o,oooo respectivamente. No entanto, as cooperativas liquidadas operam em escala mais eficiente (maior ES) com p-valor de o,ooor. Estes resultados indicam que existe uma determinada correlação entre a opção e liquidação e a ineficiência da cooperativa.

Faz-se necessário também ressalvar que, se há valores baixíssimos de eficiência técnica em retornos de escala constante e variável então haverá escores superiores de eficiência de escala, deduzindo-se por sua fórmula (veja fórmula 4). Isto implica que talvez não haja correlação entre os valores de eficiência de escala sobre sua liquidação, principalmente sobre a decisão de liquidação.

As cooperativas incorporadas se mostraram menos eficientes (foi rejeitada a hipótese nula) em termos de ETRCE frente às demais cooperativas com p-valor de o,o175. E não rejeitou a hipótese de igualdade de ETRVE e ES com p-valor de o,086o e o,2048 respectivamente.

Agrupando as cooperativas descontinuadas constatou-se que estas se mostraram menos eficientes que as demais em termos de ETRCE e ETRVE com p-valores de o,oooo e o,0219 respectivamente. E escore de eficiência escala maior com p-valor de o,oooo. Esses resultados demonstram que as cooperativas que decidem encerrar suas atividades sofreram de ineficiência técnica - sobretudo pressupondo retornos constantes.

Este resultado é importante, sobretudo em termos de política pública. A medida que o Estado queira diminuir o número de cooperativas que encerram as suas atividades o acompanhamento das medidas de eficiência técnica passa a ser uma boa Proxy para identificar a entidade que necessita ser acompanhada mais de perto.

Coelli, Estache, Perelman e Trujillo (2003) comentam mais sobre como os escores de eficiência contribuem para a implementação de políticas públicas.

E comparando as cooperativas incorporadas com as liquidadas constatou-se que as cooperativas incorporadas apresentaram maiores escores médios de ETRVE e ETRCE com p-valor de o,ooo3 e o,oo17, respectivamente. No entanto, as cooperativas liquidadas se mostraram mais eficientes em termos de escala com p-valor de o,o146. O fato de as cooperativas liquidadas apresentarem maiores escores de eficiência de escala e menores escores de eficiência técnica é intrigante e merece 
destaque, no entanto é preciso que seja feito mais estudos para identificar quais fatores que contribuem para estes resultados.

Por fim, ao considerar a ETRCE com escore superior a o,79 para definição de uma cooperativa eficiente, tem-se que dentre todas as 103 cooperativas analisadas no período de 2004 a 2014, é observado 43 cooperativas se mostraram tecnicamente eficientes em algum momento e 96 cooperativas se mostraram tecnicamente ineficientes. E nenhuma cooperativa que entrou em estado de liquidação se mostrou eficiente neste aspecto.

Com a com ETRVE, o número de eficientes aumenta para 66 e 83 se mostraram ineficientes em algum período. E três cooperativas liquidadas se mostraram eficientes. No entanto, na imagem abaixo pode-se ver que nos anos anteriores a determinação de liquidação da cooperativa não há altos níveis de ETRVE.

\section{Imagem 1 - Escores de Eficiência Técnica em Retornos Variáveis de Escala em Cooperativas liquidadas}

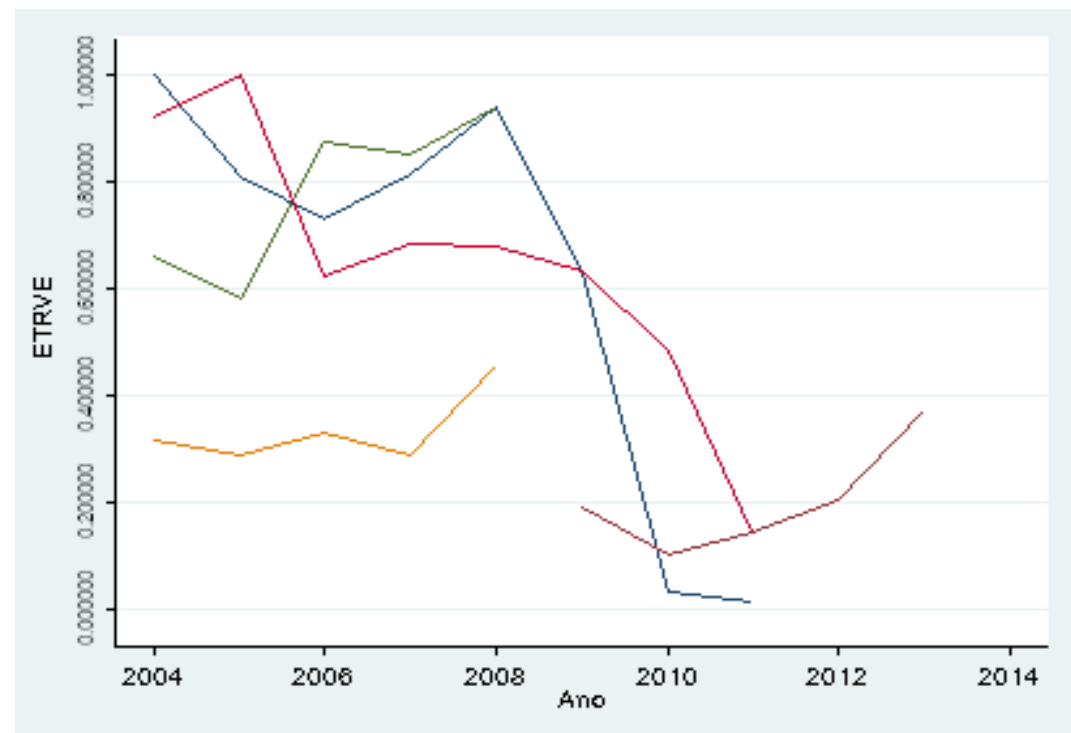

Fonte: Elaboração própria

Portanto, que o trabalho vem apontando é que a descontinuidade da cooperativa está ligada ao escore de eficiência técnica e que o destino da cooperativa após a descontinuidade está mais ligado ao tamanho do ativo. Em termos de ciclo de vida, durante a fase quatro a cooperativa tende a ser tecnicamente ineficiente e na fase cinco a escolha está mais associada ao valor da cooperativa.

\section{Conclusões finais}

A partir deste estudo pôde-se diferenciar quais fatores expressos na fase quatro tem relação sobre a fase cinco do ciclo de vida. Haja vista, as entidades que encerraram suas atividades obtiveram menores escores de eficiência técnica. Este dado é importante porque auxilia na avaliação de uma empresa em termos de risco de liquidação. Dado que a eficiência tem relação com a extinção da entidade. 
Outro ponto que merece ser evidenciado é que, aparentemente, as maiores cooperativas, dentre as que chegam à discussão sobre o futuro, tendem a não serem liquidadas, mas sim incorporadas por outras.

Considerando que a avaliação dos ativos das cooperativas é correlacionada com a decisão sobre o futuro da entidade, pode-se inferir que as cooperativas que procuram incorporar outras se preocupam com o valor dos ativos daquela a ser incorporado. O que é de se imaginar que se o valor contábil (pressupondo que no processo de encerramento das atividades são realizados testes de impairment sobre os ativos) dos ativos é maior que os passivos da entidade incorporada, então vale a pena a incorporação.

Este é o ponto chave da análise. As cooperativas menores não são incorporadas porque sua capacidade de geração de caixa é inferior que o custo de sua aquisição. Deste modo não há mais nada o que se fazer a não ser "fechar as portas". E isto explica o porquê de as cooperativas incorporadas não apresentarem patrimônio líquido negativo e apresentarem um alto grau de endividamento.

Ainda é necessário um aprofundamento no trabalho, incorporando índices financeiros e o valuation das sociedades para que as conclusões sejam tomadas com maior respaldo teórico e empírico. Também é preciso a ponderação da análise de acordo com fatores externos a cooperativa assim como o cálculo de eficiência alocativa.

\section{Referência bibliográfica}

BANKER, R. D.; CHARNES, A.; COOPER, W. W.. Some Models for Estimating Technical and Scale Inefficiencies in Data Envelopment Analysis. Austin: Management Science, v. 30, n. 9, p. 1078-1093, 1984.

BARTON, D. G. What is a Cooperative? In: COBIA, D. W. (ed.). Cooperatives in Agriculture. New Jersey: Regents/Prentice Hall, 1989. cap. 1, p. 1-20.

BIALOSKORSKI NETO, S. Economia e Gestão de Organizações Cooperativas. $2^{\underline{a}}$ ed. São Paulo: Atlas, 2012.

BRAGA, M. J.; FERREIRA, M. A. M.; DE SOUZA, U. R.. Fatores Associados à Eficiência Técnica e de Escala das Cooperativas Agropecuárias Paranaenses. Revista de Economia e Sociologia Rural, Vol.49, No. 3, 2011.

BURRES, M. J.; COOK M. L.. A Cooperative Life Cycle Framework. Working Paper, University of Missouri: Columbia, 2009.

CHADDAD, F. R.; COOK, M. L.. The economics of organization structure changes: A US perspective on demutualization. Annals of Public and Cooperative Economics, v.75, n. 4, p. 575-594, 2004.

CHADDAD, F.; ILIOPOULOS, C. Control Rights, Governance, and the Costs of Ownership in Agricultural Cooperatives. New York: Agribusiness, v. 29, p. 3-22, 2013.

CHARNES, A.; COOPER, W.W.; RHODES, E. Evaluating Program and Managerial Efficiency: an application of Data Envelopment Analysis to Program Follow Through. Management Science, v.27, n.6, p.668-697, jun. 1981.

COELLI, T.; ESTACHE, A.; PERELMAN, S.; TRUJILLO, L.. A Primer on Efficiency Measurement for Utilities and Transport Regulators. The World Bank, 2003. 
COOK, M. L.. The Future of U.S. Agricultural Cooperatives: A Neo-Institutional Approach. In: American Journal of Agricultural Economics 77, p. 1153-1159, 1995.

COOK, M. L.; ILIOPOULOS, C. Solutions To Property Rights Constraints In ProducerOwned And Controlled Organizations: Prerequisite For Agri-Chain Leadership?, In: ZIGGERS G. W.; TRIENEKENS, J. H.; ZUURBIER, P.J.P. Proceedings of the Third International Conference on Chain Management in Agri Business and Food Industry. Wageningen University Press, Wageningen, the Netherlands, 1998.

COSTA, D. R. M.. Propriedade E Decisões De Gestão Em Organizações Cooperativas Agropecuárias Brasileiras. São Paulo: Fundação Getúlio Vargas, 2010.

EVANS, L.; GUTHRIE, G.. A Dynamic Theory of Cooperatives: The Link between Efficiency and Valuation. Journal of Institutional and Theoretical Economics. Vol. 162, no. 2, p 364-383, 2006.

FERREIRA, M. A. M.. Eficiência Técnica e de Escala de Cooperativas e Sociedades de Capital na Indústria de Laticínios do Brasil. 11 de Abril de 2005. 158 f. Tese (Doutorado) - Universidade Federal de Viçosa. Viçosa, 11 de Abril de 2005.

FARREL, M. J. The Measurement of productive efficiency. Jornal of the Royal Statistical Society, Vol. 120, pp. 252-290, 1957.

FULTON, M. E.; HUETH, B.. Cooperative conversions, failures and restructurings: an overview. Journal of Cooperatives, V. 23, p 1-11, 2009.

HANSMAN, H. The Ownership of Enterprise. Cambridge: The Belknap Press of Harvard University Press, 1996.

HELMBERGER, P. G. Future roles for Agricultural Cooperatives. American Jornal of Agricultural Economics, 1966.

HIND, A. M.. Co-operative Life Cycle and Goals. Jornal of Agricultural Economic, Vol. 50, No. 3, pp. 536-548, 1999.

JENSEN, M. C.; MEKCLING, W. H.. Theory of the Firm: Managerial Behavior, Agency Costs, and Ownership Structure. Journal of Financial Economics, V. 3, No. 4, p. 305360, 1976.

JORNAL GAZETA DO POVO. Coamo compra Coagel e espera faturar 10\% mais. Disponível em: http://www.gazetadopovo.com.br/agronegocio/agricultura/coamocompra-coagel-e-espera-faturar10-mais-2ci4xwaeww876znpo88dzfqfd. Acesso em: 28.jan.2016.

JORNAL VALOR ECONÔMICO. Para avançar em café e grãos, Coopercitrus vai incorporar cooperativas em São Paulo e Minas. Disponível em: http://www.valor.com.br/agro/4341836/paraavancar-em-cafe-e-graos-coopercitrus-vaiincorporar-cooperativas-em-sao-paulo-e-minas. Acesso em: 28. Jan.2016.

MILLER R. L.. Microeconomia: Teoria, Questões e Aplicação. São Paulo: McGraw-Hill, 1981.

NILSSON, J.. Organizational principles for Cooperative firms. Scandinavian Jornal of Management, No. 17, pp. 329-356, 1999.

OBSERVATÓRIO DO COOPERATIVISMO - OBSCOOP. Balanços Patrimoniais De Cooperativas Paulistas Disponibilizados dos anos de 2007 a 2014. Ribeirão Preto, 2015. 

OCB-MS. Balanços Patrimoniais Disponibilizados dos anos de 2007 a 2014. Campo Grande, 2015.

ORGANIZAÇÃO DAS COOPERATIVAS DO ESTADO DO PARANÁ - OCEPAR. Balanços Patrimoniais Disponibilizados dos anos de 2004 a 2014. Curitiba, 2015.

PINDYCK, R. S.; RUBINFELD, D. L.. Microeconomia: Teoria Microeconômica. São Paulo: Makron Books, 1998.

PORTER, P. K.; SCULLY, G. W.. Economic Efficiency in Cooperatives. Journal of Law and Economics, Vol. 30, No. 2, p. 489-512, 1987.

PRIOR, D.; SOLÀ, M. Measuring productivity and quality changes using data envelopment analysis: an application to catalan hospitals. Financial Accountabilty \& Management, Vol. 17, No. 3, p.219-245, aug. 2001.

REVISTA CAIFEICULTURA. Cooagri, maior cooperativa de MS, é liquidada. Disponível em: www.revistacafeicultura.com.br/index.php/envia_materia.php?mat=26651. Acesso em: 28 . De jan.2016.

REVISTA GLOBO RURAL. Paulista negocia parceria com espanhóis. Disponível em:http://revistagloborural.globo.com/GloboRural/o,6993,EEC10207641931,oo.html. Acesso em: 28 de jan.2016.

SCHRADER, L. F.. Equity Capital Restructuring of Cooperatives as Investor-Owned Firms. Journal of Agricultural Cooperation 4, 1989.

SEXTON, Richard, J.; ISKOW, J.. Factors Critical to the Success or Failure of Emerging Agricultural Cooperatives. Giannini Foundation Information Series, 1988.

STAATZ, J. M.. Farmers' Incentives To Tare Collective Action Via Cooperatives: A Transaction Cost Approach. p. 87-107, 1987.

VITALIANO, P. Cooperative Enterprise: An Alternative Conceptual Basis for Analyzing a Complex Institution. American Journal of Agricultural Economics, Vol. 65, No. 5, p 10781083, 1983.

ZYLBERSZTAJN, D. Quatro Estratégias Fundamentais para as Cooperativas Agrícolas. In: BRAGA, M. J.; REIS, B. S. (org). Agronegócio Cooperativo: Reestruturação e Estratégias. Viçosa: UFV, p. 55-75, 2002. 